\section{Rapidly Growing Cutaneous Squamous Cell Carcinoma of the Nose in Chronic Lymphocytic Leukaemia}

Seth Dhillon

Cite as: Dhillon, S. Rapidly Growing Cutaneous Squamous Cell Carcinoma of the Nose in Chronic Lymphocytic Leukaemia. The Physician 2020 vol 6; issue 2: abp13 DOI: 10.38192/1.6.2.19

\begin{tabular}{l} 
Article Information \\
\hline Epub: 27.11.2019 \\
Published21.08.2020 \\
Open Access- Creative Commons Licence CC- \\
BY-ND-4.0
\end{tabular}

Poster presented at BAPIO National Conference, London Nov 2019

Peer reviewed by Subarna Chakravorty \& Sunil Daga

Keywords:

Chronic Lymphocytic leukaemia, Squamous cell carcinoma

Patients with chronic lymphocytic leukaemia (CLL) are at an increased risk of developing cutaneous squamous cell carcinoma (cSSC). In addition, the cSCCs appear to behave more aggressively, most likely due to the immunosuppressed state of the CLL patient. The literature documenting the clinical course of cSCCs in CLL is sparse.

We present a case of an exceptionally fast growing CSCC of the nose in a patient with CLL.

An 89-year old gentleman with a pre-existing diagnosis of CLL was referred to us with an CSCC of the dorsum of the nose. The tumour encompassed almost the entirety of the nose, extending to the right medial canthus.

The lesion was identified as a local recurrence of a moderately-differentiated primary cSCC which was excised 11 months previously by curettage and cautery without further follow-up.

The CSCC subsequently recurred and underwent a period of exceptionally rapid growth. In consideration of the patient's age and comorbidities it was deemed that radical surgery, requiring total rhinectomy and right orbital exenteration, was not appropriate. The patient instead received palliative radiotherapy.

The current guidelines state that any CSCC in an immunocompromised patient should be treated as high-risk and managed accordingly.

This case highlights the need for an increased awareness that CLL causes progressive immunodeficiency, hence all cSCCs in these patients should be treated as high risk. Most importantly, the chosen method of excision should allow marginal clearance to be established, significantly reducing the chance of local recurrence. The case also brings to the fore the challenges in making treatment decisions for patients nearing end of life with multiple co-morbidities.

Conflict of Interests/Comments: Nothing to declare. 